\title{
A Novel Mutation in Medium Chain Acyl-CoA Dehydrogenase Causes Sudden Neonatal Death
}

\author{
Jeffrey C. Brackett, * Harold F. Sims, ${ }^{\ddagger}$ Robert D. Steiner, ${ }^{\ddagger 5}$ Mark Nunge, ${ }^{\ddagger}$ Edward M. Zimmerman,"

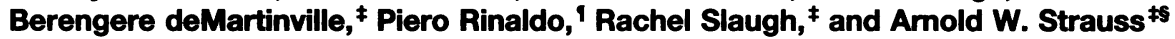 \\ Departments of $*^{*}$ Medicine and ${ }^{\ddagger}$ Pediatrics, Washington University School of Medicine, St. Louis, Missouri 63110; ${ }^{8}$ St. Louis Children’s \\ Hospital, St. Louis, Missouri 63110; "Center Medical Associates, Inc., Aliquippa, Pennsylvania 15001; and 'Department of Genetics, \\ Yale University School of Medicine, New Haven, Connecticut 06520-8005
}

\begin{abstract}
Medium chain acyl-CoA dehydrogenase (MCAD) deficiency is the most common known genetic disorder of fatty acid oxidation. Most $(\sim 80 \%)$ cases are homozygous for a single mutation: A to $G$ replacement at nucleotide 985 (A985G). MCAD deficiency typically presents in the second year of life as hypoketotic hypoglycemia associated with fasting and may progress to liver failure, coma, and death. Prompt diagnosis and management may prevent long-term sequelae.
\end{abstract}

MCAD deficiency was verified by analysis of urinary acylglycine and serum acylcarnitine species from two neonates referred for diagnosis. Full-length CDNA and MCAD exon 7 and 11 genomic clones were prepared for sequence analysis. Normal and mutant cDNAs were expressed in bacteria, and enzymatic activity was assayed by the ferricenium hexaflurophosphate method.

Four compound heterozygote individuals from two unrelated families with A985G on one allele and a novel $G$ to $A$ mutation at nucleotide 583 (G583A) as the second mutant allele presented with MCAD deficiency in the first week of life. The expressed G583A mutant protein lacks enzymatic activity.

This novel mutation, G583A, is associated with severe MCAD deficiency causing hypoglycemia or sudden, unexpected neonatal death. This previously unrecognized phenotype of MCAD deficiency may contribute significantly to preventable infant deaths. (J. Clin. Invest. 1994. 94:14771483.) Key words: fatty acid oxidation - medium chain acylCoA dehydrogenase deficiency • sudden infant death • amplification refractory mutation system • point mutation

\section{Introduction}

Medium chain acyl-CoA dehydrogenase (MCAD, EC 1.3.99.3 ${ }^{1}$ catalyzes the rate-limiting step in fatty acid oxidation

Address correspondence to Arnold W. Strauss, Division of Cardiology, St. Louis Children's Hospital, One Children's Place, St. Louis, MO 63110.

Received for publication 22 February 1994 and in revised form 2 May 1994

1. Abbreviations used in this paper: ARMS, amplification refractory mutation system; ASO, allele-specific oligonucleotide hybridization; MCAD, medium chain acyl-CoA dehydrogenase; SIDS, sudden infant death syndrome.

J. Clin. Invest.

(C) The American Society for Clinical Investigation, Inc.

0021-9738/94/10/1477/07 $\$ 2.00$

Volume 94, October 1994, 1477-1483 for intermediate (6-12 carbons) length straight-chain fatty acids. Deficiency of this enzyme is the most common defect of $\beta$-oxidation, with a predicted frequency variably estimated as 1 in 6,400-46,000 live births in populations of northern European background (1). Although there is considerable phenotypic variability, the disease typically presents in toddlers (mean age of detection, $13.5 \mathrm{mo}$ ) (2) as hypoketotic hypoglycemia. Acute presentation of the illness is precipitated by decreased caloric intake often associated with viral gastroenteritis. If not recognized promptly, the illness may progress to coma, liver failure, or death. Prompt treatment with glucose usually halts disease progression and averts long-term sequelae. Confounding the diagnosis of MCAD deficiency is the fact that this clinical presentation is quite similar to that of Reye's syndrome. Younger age at presentation, a history of unexplained sibling death, previous episodes of hypoglycemia or lethargy, and less pronounced elevations of transaminases and prothrombin time all favor the diagnosis of fatty acid oxidation defects (3).

Sudden and unexplained death, usually in the second year of life, is the presenting sign in as many as one quarter of cases of MCAD deficiency (4). Many such patients are initially diagnosed as suffering from the sudden infant death syndrome (SIDS). A family history of SIDS has prompted the retrospective diagnosis of MCAD deficiency in several instances (5).

Once MCAD deficiency is suspected, the diagnosis is confirmed by measuring levels of the metabolites hexanoylglycine, phenylpropionylglycine, and suberylglycine in the urine (6), C8-acylcarnitine and C8/C10 acylcarnitine ratios in the blood (7), or enzyme activity levels in cultured fibroblasts or white blood cells $(8,9)$. Several genetic screening tests have also been described (10-13).

A recent summary reported that $\sim 80 \%$ of MCAD-deficient patients are homozygous for a single point mutation A985G (14). Compound heterozygotes with A985G on one allele and a variety of point mutations, insertions, and deletions on the other allele account for the remainder of cases.

We recently evaluated two families with sudden unexpected neonatal deaths, originally diagnosed as SIDS. Review of the case histories suggested the possibility of a disorder in fatty acid oxidation, but genetic screening for the common mutation of MCAD deficiency proved inconclusive. The purpose of this study was to elucidate the cause of sudden death in these neonates and to reexamine the possible contribution of severe forms of MCAD deficiency and other $\beta$-oxidation defects to neonatal cardiomyopathy, fatty liver, and sudden death.

\section{Methods}

Family A. Fig. 1 illustrates this pedigree. AII-1 and AII-2 are the children of healthy, unrelated parents with no family history of MCAD deficiency or SIDS. AII-1 was a full-term, breast-fed infant (birth wt 


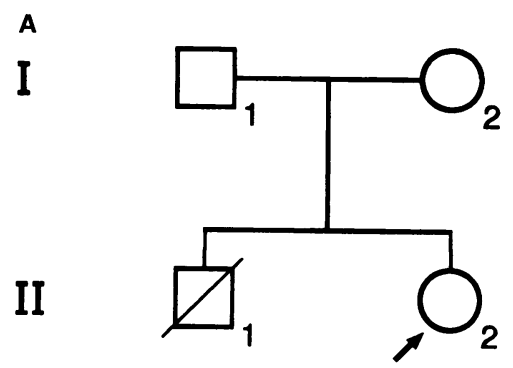

B

I

II

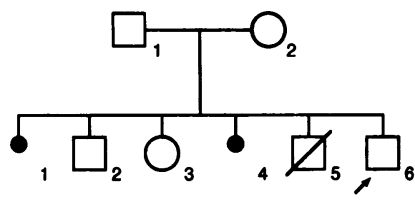

Figure 1. Pedigrees of two families with MCAD deficiency. $A$ shows the pedigree of the first family (AI-1, AI-2, etc.) referred for analysis. The index patient is indicated by the arrow, and the hash mark indicates a deceased individual. $B$ is the pedigree of the second family (BI-1, BI-2, etc.). Again the arrow indicates the index patient, and the slash mark denotes a deceased individual. The filled circles denote fetal deaths (see text).

3,010 g, Apgars 9 and 10), who was doing well until the third day of life, when he refused a feeding. Several hours later he was found dead. Autopsy revealed marked fatty infiltration of the liver with a microvesicular pattern of distribution. Kidneys and skeletal muscle were normal. The heart was harvested for valve donation and was not examined. Postmortem biochemical analysis revealed medium chain dicarboxylic aciduria, suggesting the diagnosis of MCAD deficiency.

The second child, AII-2, was delivered at term (wt 3,515 g). Delivery was complicated by a tight nuchal cord and severe birth asphyxia. Apgar score was 3 at $1 \mathrm{~min}, 6$ at $5 \mathrm{~min}$, and 8 at $10 \mathrm{~min}$. She was treated with $\mathrm{O}_{2}$, and infection was ruled out. Because MCAD deficiency was suspected in her older sibling, AII-2 was treated with frequent feedings and underwent early biochemical testing, which revealed markedly elevated levels of $n$-hexanoylglycine ( $23 \mu \mathrm{g} / \mathrm{mg}$ creatinine, $\mathrm{nl} 0.2-$ 1.9), 3-phenylpropionylglycine $(2.7 \mu \mathrm{g} / \mathrm{mg}$ creatinine, $\mathrm{nl}<1.1)$, and suberylglycine $(70 \mu \mathrm{g} / \mathrm{mg}$ creatinine, $\mathrm{nl}<11.0)$ in the urine. She has continued with frequent feedings and carnitine supplementation and has exhibited no overt symptoms since her birth.

Parental genetic testing revealed that the father, AI-1, was a heterozygote for A985G; however, the mother, AI-2 was homozygous wildtype at this locus. Both parents have normal urinary acylglycine profiles and both are of European descent.

Family B. BII-6 (Fig. 1 B) was the product of a 40 -wk gestation born without complications (birth wt, 4,075 g, Apgars 5 and 7) to unrelated parents who are both of European descent. He was well until the evening of the second day of life when he became tachypneic ( 100 breaths/min) and developed a dusky pallor. His serum glucose was 34 $\mathrm{mg} / \mathrm{dl}(1.9 \mathrm{mmol} /$ liter $)$. Infection was ruled out, and he was switched temporarily from breast milk feedings to intravenous glucose. By the twelfth day of life, he was well enough to be discharged from the hospital. Family history is significant for his mother's first trimester miscarriage with her first pregnancy (BII-1), one stillborn sibling (28 wk gestation) (BII-4), and another who died suddenly on the third day of life (BII-5). Screening of urinary metabolites of BII-6 showed elevated levels of $n$-hexanolyglycine ( $36 \mu \mathrm{g} / \mathrm{mg}$ creatinine), 3-phenylpropionylglycine $(1.8 \mu \mathrm{g} / \mathrm{mg}$ creatinine $)$, and suberylglycine $(65 \mu \mathrm{g} /$ $\mathrm{mg}$ creatinine) consistent with the diagnosis of MCAD deficiency. Individuals BII-2 and BII-3 have normal plasma acylcarnitine profiles.

Autopsy of BII-5 was initially reported as showing no abnormalities; however, pathologic review confirmed the presence of fatty infiltration of the liver, heart, and thymus.

Cell culture, RNA, and DNA isolation. Skin fibroblasts were obtained from the parents of the affected children and grown in $\alpha$-MEM supplemented with $10 \%$ fetal bovine serum and $2 \mathrm{mM}$ glutamine. RNA was isolated by the guanidinium thiocyanate method (15), and poly-(A) RNA was purified using an oligo-d(T) resin (Qiagen Inc., Chatsworth, CA). DNA was isolated from cord blood of AII-2 and from a paraffin block of liver tissue of AII-1 and BII-5. Genomic DNA from BII-2,
Table I. Oligonucleotides Used for PCR Amplification, ARMS, and ASO Screening*

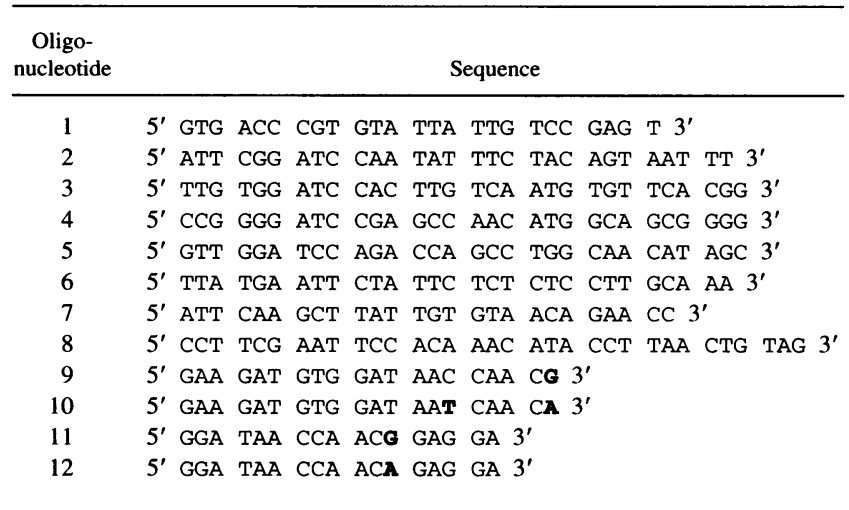

* Oligonucleotides 1 and 2 were used to amplify full-length MCAD cDNA. The products were then amplified with nested oligonucleotides 3 and 4 in preparation for subcloning. Oligonucleotides 5 and 6 were used to amplify exon 11 genomic DNA, and 7 and 8 were used to amplify exon 7 . Sense oligonucleotides 9 and 10 were used for the amplification refractory mutation system with mismatched bases shown in bold. Oligonucleotides 11 and 12 are the wild-type and mutantspecific oligonucleotides used for ASO hybridization with the base corresponding to nucleotide 583 shown in bold type.

BII-3, and BII-6 was isolated from whole blood using an alkaline lysis and proteinase digestion kit (Invitrogen, San Diego, CA) following the manufacturer's instructions. DNA was extracted from autopsy specimens as previously described (16), concentrated, and purified using Instagene (Bio-Rad Laboratories, Hercules, CA) matrix following the manufacturer's instructions.

Enzyme activity and metabolite assays. MCAD activity of mutant and normal protein expressed in bacteria was measured using ferricenium hexaflurophosphate as the electron acceptor (9). Activity was determined on duplicate samples of at least three different protein concentrations. Acylglycine and acylcarnitine measurements were performed as previously described $(6,7)$.

Cloning of MCAD cDNA and genomic DNA. Using 0.5-2 $\mu \mathrm{g}$ of total RNA (or $0.1 \mu \mathrm{g}$ of poly-(A) RNA) as template, cDNA was synthesized in the presence of $5 \mathrm{mM} \mathrm{MgCl}{ }_{2}$ per manufacturer's instructions (Perkin-Elmer Corp., Norwalk, CT). Amplification of the cDNA was performed as described previously (10) using oligonucleotides shown in Table I. The cDNA was cloned into the PGEM3Z plasmid (Promega Corp., Madison, WI), and sequence was determined by the dideoxy method.

1-2 $\mu \mathrm{g}$ of genomic DNA was amplified for 30 cycles with the following cycle program: annealing for $1 \mathrm{~min}$ at $60^{\circ} \mathrm{C}$, extension for 2 min at $72^{\circ} \mathrm{C}$, and denaturation for $1 \mathrm{~min}$ at $95^{\circ} \mathrm{C}$. The PCR products were cloned as above.

Allele-specific oligonucleotide hybridization (ASO) and amplification refractory mutation system (ARMS). Oligonucleotide probes complementary to the mutant and wild-type alleles at nucleotide 583 were prepared on an oligonucleotide synthesizer (Applied Biosystems, Inc., Foster City, CA) (Table I). Genomic DNA was amplified as above, and $0.5 \mu \mathrm{g}$ (concentration determined by $\mathrm{A}_{260}$ ) was applied to a nylon membrane (GeneScreen; New England Nuclear, Boston, MA) with a vacuum blotting device. Overnight hybridizations were done at $37^{\circ} \mathrm{C}$ in the presence of $5 \times \mathrm{SSC}, 0.02 \%$ Ficoll, $0.02 \%$ polyvinylpyrrolidone, $0.02 \%$ bovine serum albumin, $0.05 \mathrm{M}$ sodium phosphate $(\mathrm{pH} 6.8)$, $0.2 \%$ SDS, $5 \mathrm{mM}$ EDTA, $100 \mu \mathrm{g} / \mathrm{ml}$ tRNA, and $20 \mathrm{pmol}$ of ${ }^{32} \mathrm{P}$-labeled oligonucleotide. Washes were performed under the following conditions: (a) $30 \mathrm{~min}$ at $4^{\circ} \mathrm{C}$ in $3 \mathrm{M}$ tetramethylammonium chloride $/ 50 \mathrm{mM}$ Tris-Cl ( $\mathrm{pH} \mathrm{8.0)} / 2 \mathrm{mM}$ EDTA (TMA); (b) $30 \mathrm{~min}$ at $37^{\circ} \mathrm{C}$ in TMA and $0.2 \% \mathrm{SDS} ;(c) 30 \mathrm{~min}$ at $59^{\circ} \mathrm{C}$ in TMA and $0.2 \% \mathrm{SDS} ;(d) 10$ 
min at room temperature in TMA; and (e) 2 min at room temperature in $2 \times \mathrm{SSC}$ and $2 \mathrm{mM}$ EDTA. Autoradiographs exposed for $30 \mathrm{~min}$ to $2 \mathrm{~h}$ at $-70^{\circ} \mathrm{C}$ produced adequate images.

ARMS was designed to rapidly detect the novel mutation as well as the common mutation. A similar system to detect the common mutation has been described (13). PCR amplification in exon 7 using oligonucleotides differing at only the 3 ' nucleotide did not produce specific products. To increase the specificity, an additional mismatch was incorporated at the position 5 bases from the $3^{\prime}$ end of the oligonucleotides, as has been described in other systems (17). The effect of this additional change is to further destabilize the template-primer pair, as an additional mismatch is present, producing an adduct that cannot be extended by the DNA polymerase. The reactions were carried out in the presence of $1.75 \mathrm{mM} \mathrm{MgCl}$, and the cycles were as follows: annealing, $30 \mathrm{~s}$ at $56^{\circ} \mathrm{C}$; extension, $30 \mathrm{~s}$ at $72^{\circ} \mathrm{C}$; and denaturation, $60 \mathrm{~s}$ at $95^{\circ} \mathrm{C}$.

Expression of mutant protein in Escherichia coli. A mutant clone was prepared using PCR-directed mutagenesis of wild-type MCAD. This clone consisted of full-length MCAD cDNA (with no 5' or 3' untranslated sequence) in the pET-11 prokaryotic expression vector (Novagen, Inc., Madison, WI). E. coli, strain BL21(DE3), were transformed with the G583A mutant MCAD, wild-type MCAD, or pET11 vector alone. Lysates of the transformed bacteria were assayed for enzymatic activity to C8-acyl-CoA as described above.

\section{Results}

Delineation of a new MCAD mutation in family $A$. The index case (AII-2, Fig. 1) was investigated because the older sibling (AII-1) had died unexpectedly at $3 \mathrm{~d}$ of age with fatty infiltration of the liver at autopsy. Although this clinical presentation would have been very unusual for typical MCAD deficiency $(2,4)$ secondary to the common (A985G) mutation, the biochemical results performed postmortem in AII-1 and at birth in AII-2 strongly suggested the presence of MCAD deficiency. We first investigated the parents because of the ease of obtaining both abundant genomic DNA and fibroblasts. Parental genetic screening for the common MCAD mutation (A985G) with the NcoI restriction enzyme method (10-12) revealed that only the father, AI-1, was a carrier for this abnormality. The mother had the normal A at position 985 on both alleles. Thus, neither child (AII-1 or AII-2) could have the most common genotype, i.e., homozygosity for A985G, associated with MCAD deficiency. We postulated that AII-2 was a compound heterozygote, inheriting her father's A985G mutant allele as well as a different mutation from her mother.

To identify the second, uncommon mutation, full-length cDNA clones from the mother (AI-2), prepared by PCR amplification from fibroblast RNA, were subjected to complete sequence analysis. Only one difference, G583A, was present in more than one maternal cDNA clone. To confirm that this difference existed in the maternal genome, as would occur with a true mutation (as compared with a PCR-generated transcription error), maternal genomic DNA including MCAD exon 7 and $\sim 200$ bp of surrounding intron was amplified by PCR (Table I, oligonucleotides 7 and 8 ). Five subclones of this amplified DNA were analyzed by sequencing. Of these, one was wildtype, and four contained the G583A mutation, suggesting that she is a heterozygote for this novel mutation. Four exon 11 clones were also sequenced. All had the normal A at position 985 , consistent with the conclusion that neither maternal allele contained the common mutation. These results strongly suggested that the second MCAD mutation in this family was G583A in exon 7 and that the mRNA containing this mutation was stably expressed in fibroblasts. Among the known, rare
MCAD mutations (14), a change at position 583 has not been described.

Verification of the paternal (AI-1) genotype was accomplished by partially sequencing five cDNA clones after PCR amplification of his fibroblast mRNA. The common mutation (A985G) was present in one clone. A second clone had a deletion of exon 8 , an event we have observed previously in individuals homozygous or heterozygous for the A958G mutation (10). Four genomic clones from exon 7 showed no difference from the normal sequence. These results demonstrate that the father expresses the A985G mutation in mRNA, does not have other mutations expressed in mRNA, and does not have the G583A mutation on either allele, consistent with his being a simple heterozygote.

These results allowed us to focus on this new, G583A, mutation in our analysis of the offspring. After amplification of genomic DNA from the index case, AII-2, six exon 7 clones were sequenced (Fig. 2), resulting in the identification of four wild-type and two A583 mutant clones. Of the exon 11 clones, one was wild-type at position 985, and four had the A985G mutation (Fig. 2). We conclude that the living daughter (AII2 ) in this family, who is affected by biochemical criteria, is a compound heterozygote MCAD-deficient individual, with the common A985G mutation on the paternal allele and the new, G583A mutation on her maternal allele. Unfortunately, DNA of adequate quality for analysis could not be obtained from autopsy tissue of AII- 1 .

ASO screening. In preparation for large-scale screening for this new mutation to determine the frequency in the general population and for more efficient analysis of family members, we developed two strategies for screening, ASO hybridization to amplified exonic genomic DNA and ARMS with oligonucleotides for this mutation.

Performance of the ASO assay required preparation of oligonucleotide probes specific for the wild-type ( $G$ at position 583 ) and the mutant (A at position 583) alleles. Amplified exon 7 genomic DNA from normal controls, several individuals with other, enzymatically verified disorders of $\beta$-oxidation, from two individuals homozygous for the A985G mutation, from one individual heterozygous for the A985G mutation, and from family A members was analyzed with specific oligonucleotides by slot blot (Fig. 3). The G583A mutant allele was not present in the father (AI-1), an A985G homozygote with typical presentation at age $15 \mathrm{mo}$, nor in a previously reported A985G homozygote with neonatal presentation of MCAD deficiency ( slot 9 in Fig. 3) (18). As expected from the results presented above, this analysis confirmed that both the mother (AI-2) and living daughter (AII-2) are heterozygous at base 583. These results demonstrated the efficacy and reliability of this ASO screen for the new mutation.

Characterization of MCAD-deficient family $B$. As we completed the delineation of the G583A mutation in family A and developed the ASO and ARMS screening methods, family B (Fig. 1) was referred for metabolic workup secondary to sudden neonatal death in one sibling (BII-5) and a life-threatening event associated with hypoglycemia in a second sibling (BII6). Metabolic screening in individual BII-6 was consistent with MCAD deficiency. The family history, with severe illness and death in the neonatal period, bore obvious similarity to the history of our initial family. Therefore, ARMS (Fig. 4) and ASO (not shown) for our newly discovered G583A mutation were done with DNA isolated from members of family $B$. To 


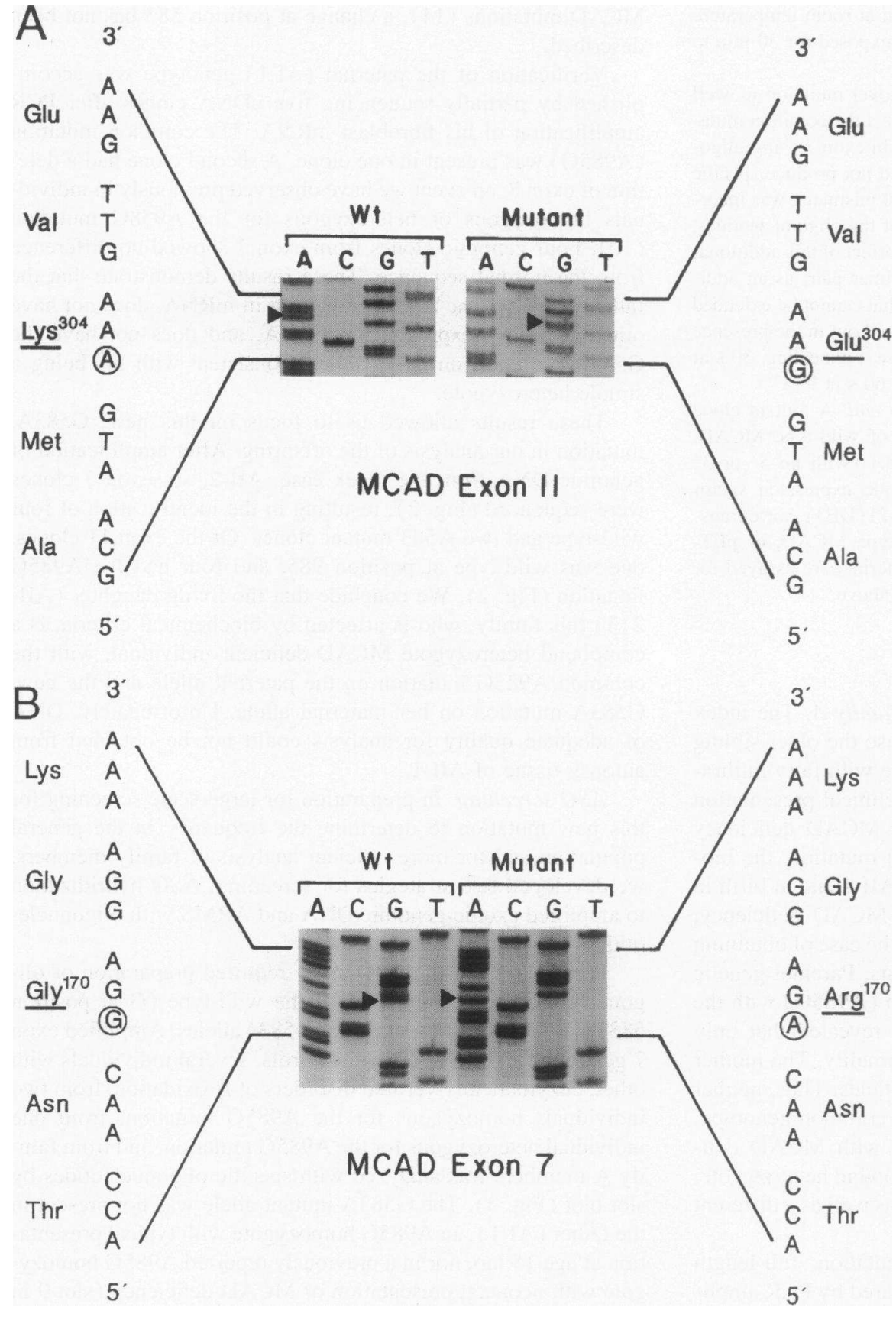

Figure 2. DNA sequence gels demonstrating the two different MCAD mutations in patient AII-2. (A) Sequence gel of two exon 11 clones from amplified genomic DNA of AII2. PCR-amplified genomic DNA was cloned into the PGEM-3Z vector for sequence analysis. The sequence was generated using the sp6 promoter primer and the dideoxy method. Two clones from the living, affected neonate from family A are shown. The mutation occurs at nucleotide 985 predicting an amino acid change from a lysine to a glutamic acid at residue 304 of the mature protein. The affected neonate is a heterozygote for this, the common MCAD mutation. $(B)$ Sequence gel of two exon 7 clones from amplified genomic DNA of AII-2. The affected neonate is heterozygous at nucleotide 583 with either the wild-type $G$ and the mutant A at this position. The mutation predicts the substitution of an arginine for glycine at residue 170 of the mature protein. our surprise, the results suggested that the proband in this family (BII-6) and three of his siblings (BII-2, BII-3, and BII-5) were heterozygous for this mutation. As in family $\mathrm{A}$, this mutation was inherited from the mother (BI-2). The results were confirmed by sequence determinations of subclones derived from PCR amplification of exon 7 in the mother (BI-2), father (BI1), living affected son (BII-6), and in the son who died on the third day of life (BII-5) (data not shown). Routine screening for the common A985G mutation by restriction digestion of exon $11 \mathrm{PCR}$ products demonstrated that the father was a carrier heterozygote and that BII-6 also carried this mutation. ASO and ARMS for the A985G mutation and sequence analyses of multiple exon 11 subclones confirmed these results. Thus, in family B, the two children (BII-5 and BII-6) who became ill or died in the neonatal period are compound heterozygotes for both the common A985G mutation and the new, exon 7 mutation (G583A). The other siblings carry a single mutant allele: G583A in BII-2 and BII-3. These data demonstrate that allelespecific PCR was able to distinguish between the two alleles in exon 7 and exon 11 of MCAD, providing a second and even simpler method for mutation screening. Correlation of clinical outcome and genotype in these two MCAD-deficient families strongly suggests that the G583A/A985G compound heterozygous state carries high morbidity and mortality in the neonatal period.

Given the severe symptoms associated with this newly defined genotype, we characterized the effect of the G583A mutation on enzymatic activity of the encoded mutant MCAD pro- 


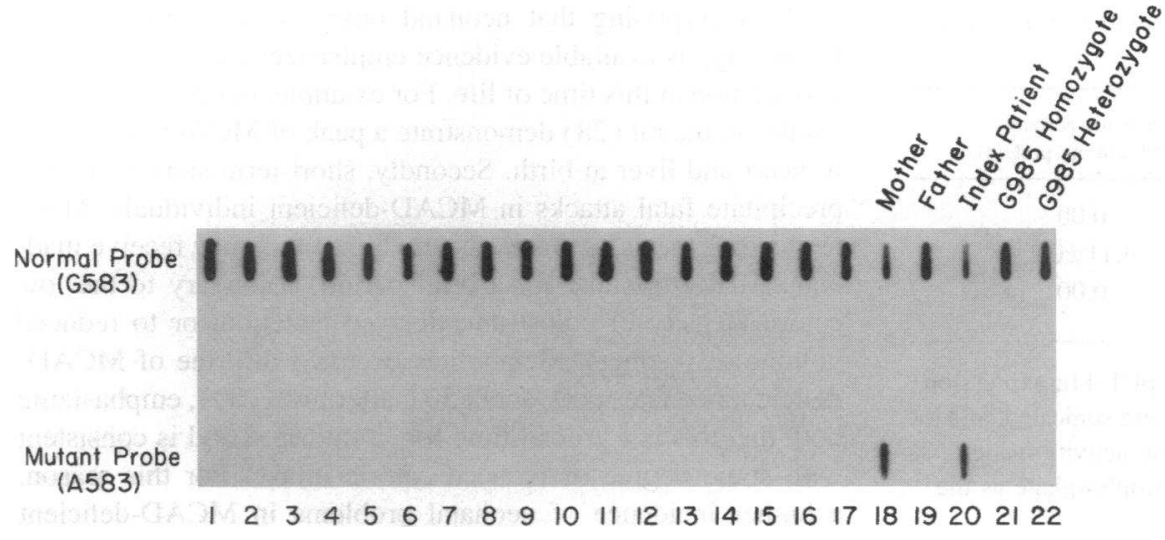

Figure 3. Detection of the G583A novel mutation by ASO hybridization. Exon 7 genomic DNA was amplified from 22 different individuals, and the resultant DNA was applied in two equal aliquots to nylon membranes. Oligonucleotides specific for the wild-type and mutant alleles were prepared and labeled with ${ }^{32} \mathrm{P}$. Unbound label was removed by washing, and autoradiography resulted in the pictured blots. Hybridization of the wild-type probe (with G583) is shown across the top, and that of the mutant probe (with A583) is shown across the bottom of the figure. Individuals 1-6 are normal controls; patients 7,8 , and $10-17$ have enzymatic assays consistent with disorders of

fatty acid oxidation other than MCAD deficiency; individuals 9 and 21 are both MCAD deficient (A985G homozygotes); and the phenotypically normal person 22 has simple heterozygosity of A985G. The mother, father, and index patient refer to AI-2, AI-1, and AII-2, respectively.

tein. Expression in bacteria of mutant and wild-type cDNA was performed. Lysates of bacteria transformed with vector alone had no detectable enzymatic activity (Table II). The wild-type construct expressed a high level of enzymatically active protein. In marked contrast, the G583A mutant protein was inactive (Table II), despite abundant expression of MCAD protein as determined by Western blot (data not shown). Thus, this mutation completely abolishes MCAD activity.

\section{Discussion}

Our results document MCAD deficiency secondary to compound heterozygosity for the common A985G mutant allele and a novel G583A mutation in two unrelated families. Correlation of this newly ascertained genotype with clinical outcome documents that this is a particularly severe phenotype. Most striking is the younger age at presentation as compared with the $80 \%$ of MCAD-deficient individuals who are homozygous for the A985G mutation. Each affected child in the two families re- ported herein was either treated presumptively or experienced life-threatening symptoms within the first $3 \mathrm{~d}$ of life. Two died. Thus, MCAD deficiency as manifested in these individuals is different from the classically described defect in both the age of onset and degree of severity.

The distinguishing aspects of this phenotype can be explained on a molecular basis. MCAD has been characterized extensively, both biochemically and structurally. The enzyme is a tetrameric flavoprotein of identical subunits encoded by a nuclear gene. The precursor protein is translated from mRNA in the cytoplasm, and an $\mathrm{NH}_{2}$-terminal transit peptide required for mitochondrial compartmentalization of the mature protein is proteolytically removed during uptake into the mitochondrial matrix. Within the matrix, chaperonin-mediated protein folding allows tetramer formation and incorporation of the flavin (19). $\mathrm{X}$-ray diffraction and crystallographic methods have been used to characterize MCAD structure to $2.4 \AA$ resolution (20). Thus, effects of naturally occurring MCAD mutants on the enzyme's structure may be predicted. The G583A mutant allele that we

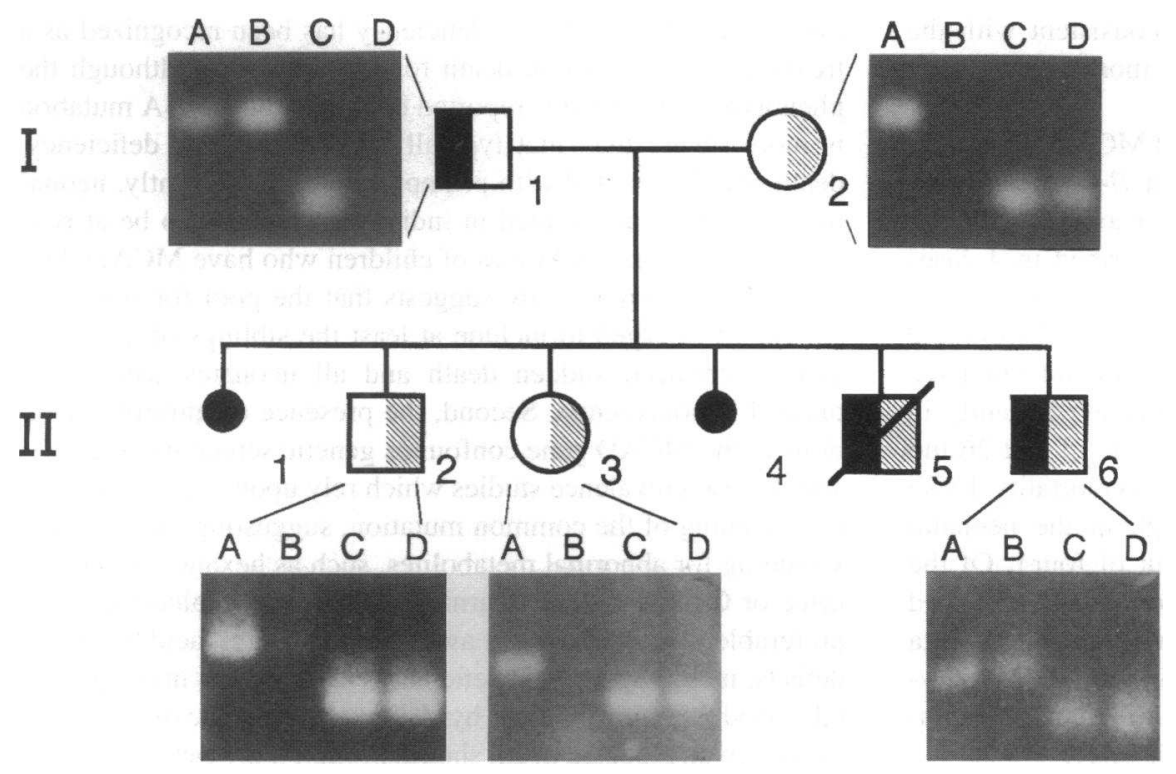

Figure 4. Delineation of MCAD mutations in family $B$ by ARMS. The family B pedigree is shown with the results of the ARMS. Lane $A$ was generated using the PCR oligonucleotide specific for the wild-type allele of exon 11. Lane $B$ represents the PCR products from amplification with the oligonucleotide specific for the mutant allele. Similarly, lanes $C$ and $D$ are the PCR products generated using the wild-type and mutant oligonucleotides for exon 7 , respectively. These data confirm that the father is a heterozygote for the common mutation with products in lanes $A$ and $B$. He is homozygous wild-type at position $\mathbf{5 8 3}$ as indicated by the presence of an amplified product in lane $C$, but not in lane $D$. Similarly, the mother is heterozygous for the novel mutation with products in lanes $C$ and $D$, but she is homozygous wild-type in exon 11 (lane $A$ ). The healthy children share their mother's genotype. The affected neonate (II6 ) is a compound heterozygote as indicated by the presence of PCR product in all four lanes. 
Table II. Activity of Wild-Type and A583G Protein Expressed in E. coli*

\begin{tabular}{lc}
\hline \multicolumn{1}{c}{ Vector } & $\begin{array}{c}\text { Specific activity } \\
(\mathrm{mM} / \mathrm{min} / \mathrm{mg} \text { protein })\end{array}$ \\
\hline pET-11a & 0.00 \\
pET-11a-MCADwt & $4.11 \pm 0.2$ \\
pET-11a-G583A-MCADmut & 0.00 \\
\hline
\end{tabular}

* Full-length MCAD cDNA was cloned into the pET-11a expression vector then transformed into $E$. coli. The cells were sonicated, and the soluble protein fraction was assayed for enzymatic activity using C:8coenzyme $\mathrm{A}$ as substrate and ferricenium hexaflurophosphate as the electron acceptor.

have discovered encodes a protein that, after expression in bacteria, is devoid of enzymatic activity. The mutation predicts a change from glycine, a neutral amino acid with no side chain, to arginine, a positively charged residue with a bulky side chain, at amino acid 195 of the precursor protein (residue 170 of the mature protein). This amino acid is conserved as a small neutral amino acid (glycine or alanine) in every known acyl-CoA dehydrogenase. Based on structural data $(20,21)$, this residue is close to the flavin-binding site. Computer modeling predicts that threonine-168 forms a hydrogen bond with the flavin adenine dinucleotide ring and that methionine-165 and tryptophan-166 overlie the sinister side of the flavin adenine dinucleotide ring and may provide a hydrophobic pocket for the flavin and allow interaction with the electron transfer flavoprotein. A radical substitution, G170R, in the vicinity of these important residues may be sufficient to disrupt flavin binding or to interfere with electron transfer. Either result would likely completely inactivate the enzyme. However, our patients are compound heterozygotes producing mRNAs from both mutant alleles. We have not determined whether patients' cells generate K304E homotetramers, G170R homotetramers, or various combinations of heterotetramers. The common K304E mutation interferes with tetramer formation (22), although tetramers which are formed have a normal specific activity (23). Further cell biological studies will be necessary to determine how these two mutations interact at the protein level in our patients. It is clear, however, that the G170R mutant MCAD is totally inactive, consistent with the severe neonatal onset and associated high mortality and low MCAD activity in fibroblasts.

Neonatal onset of symptoms with typical MCAD deficiency is extremely rare, although other defects in $\beta$-oxidation may become manifest in this period (24). In fact, neonatal presentation of MCAD deficiency has only been described in 4 cases of the more than 300 reported $(18,25-27)$ (Review of urinary acylglycine excretion [Rinaldo, P., unpublished data] from one of these [27] patients indicated that this child did not have MCAD deficiency.). Although death does occur frequently in MCAD deficiency, it is more common between 15 and 26 mo (mortality 59\%) than in infants under 12 mo (mortality $13 \%$ ) (4). The incidence of sudden death is high in the neonatal period among the published cases (three out of four). Of the four reported individuals, one was homozygous for A985G, and the genotype was not reported in the other three. These data suggest that neonatal death is uncommon in A985G homozygotes. Even with inclusion of our patients with this novel mutation, a neonatal onset is rare in MCAD deficiency.
It is surprising that neonatal onset is not reported more frequently, as available evidence emphasizes the importance of $\beta$-oxidation at this time of life. For example, our developmental studies in the rat (28) demonstrate a peak of MCAD expression in heart and liver at birth. Secondly, short-term starvation may precipitate fatal attacks in MCAD-deficient individuals. Many neonates, especially those who are breast fed, may receive inadequate caloric intake for a brief period, secondary to the low caloric density of colostrum, delayed lactation, or to reduced volume of feedings. Metabolites excreted in urine of MCADdeficient neonates peak about $50 \mathrm{~h}$ after birth (29), emphasizing both that this is a crucial time for $\beta$-oxidation and is consistent with some degree of reduced caloric intake. For this reason, a higher incidence of neonatal problems in MCAD-deficient individuals might be expected.

Because MCAD deficiency has been reported rarely in the neonate, the diagnosis may frequently be missed. In a recent retrospective analysis from Australia (30), the hospital records of 16 children with documented MCAD deficiency were reviewed. Six of the children had significant perinatal hypoglycemia (onset $17 \mathrm{~h}-3 \mathrm{~d}$ ), necessitating the administration of intravenous glucose. MCAD deficiency was not recognized in these individuals despite the presence of symptoms. Our patient, BII6 , also had neonatal hypoglycemia. In our patients (AII-1 and BII-5), a diagnosis of $\beta$-oxidation defect was not initially considered, even though autopsies revealed severe tissue steatosis, indicating that increased awareness among pathologists, as well as pediatricians, is needed. We and others $(5,31,32)$ have proven that, in some cases, individuals carrying an autopsy diagnosis of SIDS, especially those with hepatic or cardiac steatosis, did have MCAD deficiency. However, molecular analyses of SIDS patients for the A985G mutation have suggested that this allele is not overrepresented in these populations (33). These considerations emphasize that neonatal hypoglycemia may be more commonly due to inherited defects in $\beta$-oxidation, including MCAD deficiency, than previously recognized and raise the possibility that such $\beta$-oxidation defects may account for some unexplained, sudden neonatal deaths, especially if tissue steatosis is observed postmortem.

Our discovery of the severe neonatal form of MCAD deficiency secondary to a novel mutation in the MCAD gene highlights several important principles relating to fatty acid oxidation defects. First, MCAD deficiency has been recognized as a treatable cause of infant death for several years. Although the phenotype of the infants reported here with the G583A mutation is more severe than that typically seen in MCAD deficiency, death may be averted with prompt treatment. Currently, neonatal screening is performed in individuals deemed to be at risk, such as first degree relatives of children who have MCAD deficiency. Our current study suggests that the pool for screening should be enlarged to include at least the siblings of any child that experienced sudden death and all neonates with unexplained hypoglycemia. Second, the presence of multiple mutations in the MCAD gene confounds genetic screening strategies and disease prevalence studies which rely upon genetic molecular screening of the common mutation, suggesting that neonatal screening for abnormal metabolites, such as hexanoylglycine in urine or C8 and C10-acylcarnitines on neonatal blood spots, is preferable. Third, increased awareness that fatty acid oxidation defects, including MCAD deficiency, may present in the perinatal period with unexplained hypoglycemia, cardiac dysfunction, or sudden unexpected death should prompt more rapid diagnosis 
and help prevent morbidity and mortality. Finally, this report and others demonstrate that defects in fatty acid oxidative enzymes are the cause of an expanding variety of disease phenotypes, including sudden death, cardiomyopathy, and hepatic steatosis (including that associated with pregnancy [34]), which may become manifest from the neonatal period to adulthood.

\section{Acknowledgments}

The authors would like to express their appreciation for the contributions of Dr. Cheryl Coffin and Dr. David Millington to this study.

This work was supported by National Institutes of Health grants HL-07081 (J. C. Brackett) and R37-DK20407 (A. W. Strauss).

\section{References}

1. Matsubara, Y., K. Narisawa, K. Tada, H. Ikeda, Y. Ye-Qi, D. M. Danks A. Greene, and E. R. B. McCabe. 1991. Prevalence of K329E mutation in MCAD gene determined from Guthrie cards. Lancet. 338:552-553.

2. Touma, E. H., and C. Charpentier. 1992. MCAD deficiency. Arch. Dis. Child. 67:142-145.

3. Treem, W. R., C. A. Witzleben, D. A. Piccoli, C. A. Stanley, D. E. Hale, P. M. Coates, and J. B. Watkins. 1986. Medium-chain and long-chain acyl CoA dehydrogenase deficiency: clinical, pathologic and ultrastructural differentiation from Reye's syndrome. Hepatology. 6:1270-1278.

4. Roe, C. R., and P. M. Coates. 1989. Acyl-CoA dehydrogenase deficiencies In The Metabolic Basis of Inherited Disease. C. R. Scriver, A. L. Beaudet, W. S Sly, and D. Valle, editors. McGraw-Hill Inc., New York. 889-914.

5. Kelly, D. P., D. E. Hale, S. L. Rutledge, M. L. Ogden, A. J. Whelan, Z. Zhang, and A. W. Strauss. 1992. Molecular basis of inherited MCAD deficiency causing sudden child death. J. Inherited Metab. Dis. 15:171-180.

6. Rinaldo, P., J. J. O'Shea, P. M. Coates, D. E. Hale, C. A. Stanley, and K. Tanaka. 1988. MCAD deficiency. Diagnosis by stable-isotope dilution measurement of urinary $n$-hexanoylglycine and 3-phenylpropionylglycine (published erratum appears in N. Engl. J. Med. 320:1227). N. Engl. J. Med. 319:1308-1313.

7. Van Hove, J. L., W. Zhang, S. G. Kahler, C. R. Roe, Y.-T. Chen, N. Terada, D. H. Chace, A. K. Iafolla, J.-H. Ding, and D. S. Millington. 1993 MCAD deficiency: diagnosis by acylcarnitine analysis in blood. Am. J. Hum. Genet. 52:958-966.

8. Coates, P. M., D. E. Hale, C. A. Stanley, B. E. Corkey, and J. A. Cortner 1985. Genetic deficiency of MCAD: studies in cultured skin fibroblasts and peripheral mononuclear leukocytes. Pediatr. Res. 19:671-676.

9. Lehman, T. C., D. E. Hale, A. Bhala, and C. Thorpe. 1990. An acylcoenzyme A dehydrogenase assay utilizing the ferricenium ion. Anal. Biochem. 186:280-284.

10. Kelly, D. P., A. J. Whelan, M. L. Ogden, R. Alpers, Z. Zhang, G. Bellus, N. Gregersen, L. Dorland, and A. W. Strauss. 1990. Molecular characterization of inherited MCAD deficiency. Proc. Natl. Acad. Sci. USA. 87:9236-9240.

11. Matsubara, Y., K. Narisawa, S. Miyabayashi, K. Tada, P. M. Coates, C. Bachmann, L. J. Elsas II, R. J. Pollitt, W. J. Rhead, and C. R. Roe. 1990. Identification of a common mutation in patients with MCAD deficiency. Biochem. Biophys. Res. Commun. 171:498-505.

12. Yokota, I., P. M. Coates, D. E. Hale, P. Rinaldo, and R. Tanaka. 1991 Molecular survey of a prevalent mutation, A985G transition, and identification of five infrequent mutations in the MCAD gene in 55 patients with MCAD deficiency. Am. J. Hum. Genet. 49:1280-1291.
13. Tsai, M. Y., K. Schwichtenberg, and M. Tuchman. 1993. Laboratory diagnosis of MCAD deficiency by the ARMS. Clin. Chem. 39:280-283.

14. Tanaka, K., I. Yokota, P. M. Coates, A. W. Strauss, D. P. Kelly, Z. Zhang, N. Gregersen, B. S. Andresen, Y. Matsubra, D. Curtis, and Y.-T. Chen. 1992. Mutations in the MCAD gene. Human Mutation. 1:271-279.

15. Chirgwin, J. M., A. E. Przybyla, R. J. MacDonald, and W. J. Rutter 1979. Isolation of biologically active ribonucleic acid from sources enriched with ribonuclease. Biochemistry. 18:5294-5299.

16. Goelz, S. E., S. R. Hamilton, and B. Vogelstein. 1985. Purification of DNA from formaldehyde fixed and paraffin embedded human tissue. Biochem. Biophys. Res. Commun. 130:118-126.

17. Newton, C. R., A. Graham, L. E. Heptinstall, S. J. Powell, C. Summers, N. Kalsheker, J. C. Smith, and A. F. Markham. 1989. Analysis of any point mutation in DNA, the ARMS. Nucleic Acids Res. 17:2503-2516.

18. Leung, K.-C., J. W. Hammond, S. Chabra, K. H. Carpenter, M. Potter, and B. Wilcken. 1992. A fatal neonatal case of MCAD deficiency with homozygous A $\rightarrow$ G985 transition. J. Pediatr. 121:965-968.

19. Saito, T., W. J. Welch, and K. Tanaka. 1994. Intramitochondrial folding and assembly of MCAD. J. Biol. Chem. 269:4401-4408.

20. Kim, J. P., and J. Wu. 1988. Structure of the MCAD from pig liver mitochondria at 3[A] resolution. Proc. Natl. Acad. Sci. USA. 85:6677-6681.

21. Kim, J. P., M. Wang, and R. Paschke. 1993. Crystal structures of MCAD from pig liver mitochondria with and without substrate. Proc. Natl. Acad. Sci. USA. 90:7523-7527.

22. Yokota, I., T. Saijo, J. Vockley, and K. Tanaka. 1992. Impaired tetramer assembly of variant MCAD with a glutamate or aspartate substitution for lysine 304 causing instability of the protein. J. Biol. Chem. 267:26004-26110.

23. Whelan, A. J., A. W. Strauss, D. E. Hale, N. J. Mendelsohn, and D. P. Kelly. 1993. Expression and characterization of human mutant E304 MCAD in mammalian cells. Pediatr. Res. 34:694-697.

24. Duran, M., R. J. A. Wanders, J. P. deJager, L. Dorland, L. Bruinvis, D Ketting, L. Ijlst, and F. J. van Sprang. 1991. 3-Hydroxydicarboxylic aciduria due to long-chain 3-hydroxyacyl-coenzyme A deficiency associated with sudden neonatal death: protective effect of medium-chain triglyceride treatment. Eur. $J$. Pediatr. 150:190-195.

25. Catzeflis, C., C. Bachmann, D. E. Hale, P. M. Coates, U. Wiesmann, J. P. Colombo, F. Joris, and G. Deleze. 1990. Early diagnosis and treatment of neonatal MCAD deficiency: report of two siblings. Eur. J. Pediatr. 149:577-581.

26. Walker, V., G. A. Mills, G. P. Weavind, M. A. Hall, and P. G. B. Johnston 1990. Diagnosis of MCAD deficiency in an asymptomatic neonate. Ann. Clin. Biochem. 27:267-269.

27. Nobukuni, Y., T. Yokoo, Y. Ohtani, F. Endo, S. Aoki, M. Yoshinaga, T Matsumoto, M. Yoshimoto, Y. Tsuji, and I. Matsuda. 1988. Neonatal onset of MCAD deficiency in two siblings. Brain \& Dev. 10:129-134.

28. Kelly, D. P., J. I. Gordon, R. Alpers, and A. W. Strauss. 1989. The tissuespecific expression and developmental regulation of two nuclear genes encoding rat mitochondrial proteins. J. Biol. Chem. 264:18921-18925.

29. Walker, V., G. A. Mills, and M. Radford. 1990. Diagnosis of MCAD deficiency in the newborn. Lancet. 335:1288-1289.

30. Wilcken, B., K. H. Carpenter, and J. Hammond. 1993. Neonatal symptoms in MCAD deficiency. Arch. Dis. Child. 69:292-294.

31. Howat, A. J., M. J. Bennett, S. Variend, L. Shaw, and P. C. Engel. 1985 Defects of metabolism of fatty acids in the sudden infant death syndrome. $\mathrm{Br}$. Med. J. 290:1771-1773.

32. Ding, J. H., C. R. Roe, A. K. Iafolla, and Y.-T. Chen. 1991. MCAD dehydrogenase deficiency and sudden infant death. N. Engl. J. Med. 325:61-62.

33. Lundemose, J. B., N. Gregersen, S. Kolvraa, B. N. Pedersen, M. Gregersen, K. Helweg-Larsen, and J. Simonsen. 1993. The frequency of a disease-causing point mutation in the gene coding for MCAD in sudden infant death syndrome. Acta Pediatr. 82:544-546.

34. Treem, W. R., P. Rinaldo, D. E. Hale, C. A. Stanley, D. S. Millington, J. S. Hyams, S. Jackson, and D. M. Turnbull. 1994. Acute fatty liver of pregnancy and LCHAD deficiency. Hepatology. 19:339-345. 